\title{
Effects of oxybutynin transdermal system on health-related quality of life and safety in men with overactive bladder and prostate conditions
}

\author{
D. R. Staskin, ${ }^{1}$ M. T. Rosenberg, ${ }^{2}$ N. V. Dahl, ${ }^{3}$ P. V. Polishuk, ${ }^{4}$ N. R. Zinner ${ }^{5}$ \\ Linked Comment: MacDiarmid. Int J Clin Pract 2008; 62: 3-4. \\ OnlineOpen: This article is available free online at www.blackwell-synergy.com
}

\section{SUMMARY}

Aims: Overactive bladder (OAB) is common in men and may exist concomitantly with benign prostatic hyperplasia (BPH) and obstruction. We present a subanalysis of results from men with $O A B$ in a 6-month, open-label study of treatment with the oxybutynin transdermal system (OXY-TDS). Broad entry criteria were incorporated to yield a clinically representative population. Methods: All participants received OXY-TDS $3.9 \mathrm{mg} /$ day. Effectiveness was assessed by changes in scores on validated questionnaires, which included the single-item Patient Perception of Bladder Condition (PPBC), the King's Health Questionnaire (KHQ) and the Beck Depression Inventory-II (BDI-II). Results: The proportion of men $(n=369$; mean age $=$ 69.6 years) who reported that their bladder condition caused moderate, severe or many severe problems ( $P P B C \geq 4$ ) improved from $77.3 \%$ at baseline to $38.1-53.6 \%$ in subsequent months. Mean KHQ scores decreased significantly ( $p \leq 0.0196)$ from baseline to study end in eight of 10 domains, indicating improved health-related quality of life. The proportion of men with BDI-II score $>12$ (associated with a diagnosis of depression) decreased from $23.9 \%$ to $17.9 \%$ $(p=0.0055)$. Men with a history of 'prostate problems' or use of 'BPH medication' $(32.2 \%)$ had KHQ domain changes that were similar $(p \geq 0.1016)$ to those of other men. Most men (76.2\%) reported no treatment-related adverse events; two men $(0.5 \%)$ experienced symptoms of mild urinary retention, but neither required catheterisation. Conclusions: Oxybutynin transdermal system treatment of men with $O A B$ was effective and well tolerated, regardless of history of prostate condition.

\section{Introduction}

The overall prevalence of overactive bladder (OAB) in the USA is similar in men (16.1\%) and women (16.9\%) but increases with age and may become higher in men than in women after 75 years of age $(1,2)$. Benign prostatic hyperplasia and obstruction (BPH and $\mathrm{BPO}$ ) are conditions that may coexist in men and potentially complicate $\mathrm{OAB}$ treatment, and that are more common in older age groups (3). OAB and $\mathrm{BPH}$ are known to impair health-related quality of life (HRQoL) and to increase symptoms of depression (2,4-6). Studies of OAB treatments have focused primarily on patients with urinary incontinence (UI), most of whom are women (2). As a result, men have been under-represented in studies of treatment for $\mathrm{OAB}$, in which they typically constitute $<15 \%$ of the population.
What's known
- Combined treatment of men with and without $\mathrm{BPH}$ is an evolving paradigm.
What's new
- This article contributes significant safety data, from the largest study to date, in a community use situation, where anticholinergics are commonly used.
- The study provides significant quality of life benefit data in a large population.
- The community usage design did not employ inclusion or exclusion criteria that would restrict the primary care physician from administrating the medication in a "real life" setting.

It is well established that orally administered antimuscarinic drugs are effective in reducing symptoms and improving HRQoL in patients with OAB, including those who are male (7-10). However, patients with $\mathrm{OAB}$ often are prescribed medications intended to treat $\mathrm{BPH}$ (11). Furthermore, antimuscarinic drugs are rarely prescribed together with $\mathrm{BPH}$ medications, perhaps because of concern that their anticholinergic effects may exacerbate obstructive symptoms (11). Patients often discontinue therapy with orally administered antimuscarinic medications because of systemic anticholinergic effects, such as dry mouth $(12,13)$.

Guidelines from the 6th International Consultation on New Developments in Prostate Cancer and Prostate Diseases recommend antimuscarinic therapy for some older men with lower urinary tract symptoms (LUTS) (14). The guidelines on basic management
${ }^{1}$ Weill Medical College of Cornell University, New York, NY, USA

${ }^{2}$ Mid-Michigan Health Centers, Jackson, MI, USA ${ }^{3}$ Watson Laboratories, Morristown, NJ, USA ${ }^{4}$ Palomar Urology, Escondido, CA, USA ${ }^{5}$ Western Clinical Research, Torrance, CA, USA

\section{Correspondence to:} David R. Staskin, MD, New York Presbyterian Hospital-Cornell, 525 East 68th Street, F-918, New York, NY 10021, USA

Tel.: + 12127465414 Fax: + 12127468240 Email:

das2021@med.cornell.edu

Disclosures

David R. Staskin: Consultant or speaker for Astellas, Allergan, Esprit, Pfizer and Watson; study investigator for Allergan, Esprit and Watson. Matt T. Rosenberg: Consultant for Astellas Pharma Inc.

GlaxoSmithKline, Novartis Pharmaceuticals Corporation, Ortho-McNeil Pharmaceutical, Pfizer Inc. and Verathon Inc.; received fees from Esprit Pharma, Inc. and Ortho-McNeil Pharmaceutical for non-CME services; conducted research on behalf of Sanofi-Aventis. Naomi V. Dahl: Employee of study sponsor (Watson). Paul V. Polishuk: None. Norman R. Zinner: On Speaker and Advisory boards for Watson, Esprit and Astellas; conducted clinical trials for Watson, Esprit, Astellas, GlaxoSmithKline, Pfizer and Lilly.

As Urology Section Editor for the Journal, Matt T. Rosenberg withdrew from the review process and deferred all editorial decisions to Graham Jackson. 
This work was presented, in part, in a poster at the Annual Scientific Meeting of the American Geriatrics Society, 2-6 May, 2007 in Seattle, WA, USA.

Re-use of this article is permitted in accordance with the Creative Commons Deed, Attribution 2.5, which does not permit commercial exploitation. suggest fluid restriction, lifestyle modification and bladder training (14). For patients with persistent, bothersome LUTS after basic management, suggested approaches for specialised management of $\mathrm{OAB}$ and bladder outlet obstruction (BOO) include both pharmacological therapy and surgical treatment (14). The direction of medical therapy depends on symptoms and the results of specific tests. If patients have mixed $\mathrm{OAB}$ and $\mathrm{BOO}$, it is recommended that they be treated with antimuscarinics and $\alpha_{1 \mathrm{~A}}$-adrenergic receptor antagonists ( $\alpha$-blockers) (14). When BOO is the predominant condition, $\alpha$-blockers are recommended if patients have a small gland or a low $(<1.5 \mathrm{ng} / \mathrm{ml})$ prostate-specific antigen (PSA) level, but both $\alpha$-blockers and $5 \alpha$-reductase inhibitors (5-ARI) should be prescribed if patients have an enlarged gland or a higher $(>1.5 \mathrm{ng} / \mathrm{ml})$ PSA level (14).

Several studies have suggested that antimuscarinics and $\alpha$-blockers can be used concurrently in men with $\mathrm{OAB}$ and $\mathrm{BOO}$ or $\mathrm{BPH}$. A randomised, controlled efficacy and safety study of extended-release tolterodine (TOL-ER) and tamsulosin treatment in men $(N=879)$ with LUTS, including OAB, concluded that combination therapy was more efficacious than monotherapy and had similar tolerability (15). Lee et al. (16) studied men $(N=144)$ with LUTS who presented consecutively to a single tertiary care centre. Patients were classified into two groups: one in which patients had BOO alone (76/144; 53\%), and a second group in which patients had BOO plus OAB $(68 / 144 ; 47 \%)(16)$. OAB was defined as involuntary detrusor contractions at $\geq 10 \mathrm{~cm} \mathrm{H}_{2} \mathrm{O}$ (16). Improvement was defined as at least a three-point reduction in International Prostate Symptom Score (IPSS) (16). In the group with $\mathrm{BOO}$ plus $\mathrm{OAB}$, only $35 \%(24 / 68)$ of men treated with doxazosin alone reported improvement in symptoms at the end of the initial 3 -month period (16). The response rate in this group increased to $82 \%(56 / 68) 2$ months after $2 \mathrm{mg}$ tolterodine twice daily was added to doxazosin therapy (16).

An alternative to oral administration for antimuscarinic medication, if its use resulted in a lower incidence of dry mouth and other anticholinergic adverse effects, might be attractive to patients. The oxybutynin transdermal system (OXY-TDS; Oxytrol $^{\circledR}$, Watson Laboratories, Morristown, NJ) has been shown to be as effective as orally administered oxybutynin immediate release (OXY-IR) and TOLER in reducing symptoms of $\mathrm{OAB}(17,18)$, but OXY-TDS is associated with a lower rate of dry mouth $(17,18)$. Inhibition of saliva production during treatment with oxybutynin, a commonly used antimuscarinic $\mathrm{OAB}$ drug, appears to be correlated with plasma concentrations of the metabolite $\mathrm{N}$-desethyloxybutynin (19). Delivery of oxybutynin directly through the skin with OXY-TDS avoids the first-pass hepatic metabolism that occurs with orally administered oxybutynin (19). As a result, the incidence of anticholinergic adverse events with OXY-TDS therapy is similar to that with placebo transdermal treatment $(18,20,21)$.

The Multicenter Assessment of TRansdermal therapy In overactive bladder with oXybutynin (MATRIX) study was undertaken to examine the effect of OXY-TDS therapy on HRQoL and safety in a large, diverse population. Here, we present a planned analysis of results from the cohort of men who participated in MATRIX, along with a post hoc analysis of men with and without pre-existing prostate conditions, to examine the safety and effectiveness of OXY-TDS treatment for patients with OAB.

\section{Methods}

\section{Participant selection criteria}

The MATRIX (study OXY0402; registered at ClinicalTrials.gov as NCT00224146) was an open-label, prospective, randomised, multicentre cohort study of community-based adults with $\mathrm{OAB}$. The study design and main results, as well as data on sexual function, have been published elsewhere $(22,23)$. Inclusion criteria consisted of one or more symptoms of $\mathrm{OAB}$, as described on the OXY-TDS label (urge UI, urgency or frequency), age 18 years or older, and the ability to complete questionnaires without assistance. Exclusion criteria included any of the following current conditions: uncontrolled narrow-angle glaucoma, urinary retention or a treatable condition besides $\mathrm{OAB}$ that could cause UI or urgency (urinary tract infection, bladder stone, bladder tumour, prostatitis and prostate cancer). Participants could not have experienced hypersensitivity to oxybutynin or other components of OXY-TDS and could not have received prior treatment with OXY-TDS; they also could not be residents in a nursing home or a long-term care facility.

No exclusion criteria were based on maximum age, comorbid conditions besides those already described, or use of concurrent medications except other OAB treatments (or any investigational product within the previous 30 days). Participants could be enrolled despite current use of BPH medication or a history of prostate cancer. Written, informed consent was obtained from each individual before enrolment. MATRIX was conducted in accordance with the Declaration of Helsinki and Good Clinical Practice guidelines. The study protocol and procedures were approved by a central institutional review board (Independent Review Consulting, Corte 
Madera, CA) or, at a small number of study centres, by site-specific institutional review boards.

\section{Treatment}

All participants received OXY-TDS at the US Food and Drug Administration-approved dosage of $3.9 \mathrm{mg} /$ day (two patches per week) for up to 6 months. Study centres were randomised $1: 1$ within each investigator specialty (Primary Medicine, Obstetrics-Gynecology, Urology, Geriatrics or other) to provide to participants standard instructions for OXY-TDS or standard instructions plus a packet of enhanced $\mathrm{OAB}$ educational materials. Assistance with applying the first patch was provided at the enrolling centre; for subsequent applications, participants were instructed to rotate the position of patch application between the abdomen, hip and buttock. Participants returned to the clinic for visits after 1,3 and 6 months of treatment.

\section{Participant assessment of treatment effectiveness}

Participants rated their global OAB severity with use of the Patient Perception of Bladder Condition (PPBC), a validated, single-item questionnaire (24). Participants rated the difficulties caused by their bladder condition on the following six-point scale: 'no problems at all' (one), 'very minor problems' (two), 'minor problems' (three), 'moderate problems' (four), 'severe problems' (five) and 'many severe problems' (six).

Patient Perception of Bladder Condition assessments were made at the baseline clinic visit and were included during subsequent monthly scripted computer-assisted telephone interviews (CATI).

\section{Assessment of health-related quality of life impairment}

Data on impairment of HRQoL were acquired through use of the King's Health Questionnaire ${ }^{\circledR}$ (KHQ), which was administered in the clinic at baseline, 3 months and 6 months. For participants who withdrew from the study before 6 months, the KHQ and other questionnaires were administered at the time of discontinuation. The KHQ is a 27 -item, validated instrument that is used to assess symptoms and impairment of HRQoL in individuals with OAB or other lower urinary tract conditions (25). Although the KHQ was originally designed for use with women, it was subsequently validated for use in men (26). Results are converted to scores that pertain to 10 domains, with a score of zero corresponding to the least HRQoL impairment and a score of 100 signifying the worst HRQoL impairment. The minimal clinically important decrease (improvement) for most domain scores is considered to be five points; a decrease of three points is considered important for the domains of General Health Perception and Symptom Severity (27).

\section{Assessment of symptoms of depression}

Information related to depression was obtained with the Beck Depression Inventory-II ${ }^{\circledR}$ (BDI-II), a validated 21-item questionnaire that was used to assess the existence and severity of symptoms associated with depression that occurred over the preceding 2 weeks (28). Each questionnaire item contains a list of statements about a particular symptom of depression, arranged in order of increasing severity; these items are consistent with the Diagnostic and Statistical Manual of Mental Health Disorders, Fourth Edition. BDI-II summary scores can range from zero (least depression) to 63 (greatest depression); a score $>12$ is associated with a clinical diagnosis of depression.

\section{Participant-reported satisfaction with treatment}

Participants were queried about their perceptions of the OXY-TDS patch during the monthly, scripted CATI sessions. Those who had been treated previously for $\mathrm{OAB}$ were asked to compare characteristics of OXY-TDS with those of their prior therapy.

\section{Assessment of safety}

Safety was assessed by reports of adverse events recorded at postbaseline clinic visits (at 1, 3 and 6 months), or as they were reported at any time during the study. Investigators categorised adverse events by severity (mild, moderate or severe) and by possible causal relationship (related or not related) to treatment.

\section{Statistical analysis}

To provide sufficient statistical power for analyses of patient subgroups, the target population size was set at approximately 2500 participants. Analyses of effectiveness were performed with the intent-to-treat population, which comprised all participants who received at least two doses of OXY-TDS and underwent at least one postbaseline assessment. The primary effectiveness end-point in MATRIX was mean change in KHQ domain scores from baseline to study end; analyses of select KHQ item responses also are presented here. A post hoc analysis of KHQ results was conducted for men with and without pre-existing prostate problems. Secondary effectiveness end-points included self-reported changes in global $\mathrm{OAB}$ condition (PPBC score), mean change from baseline to study end in BDI-II summary score, 
and participant perceptions of the OXY-TDS patch. Safety was analysed for all participants who received at least one dose of OXY-TDS.

The significance of differences between groups in continuous variables was determined by analysis of co-variance (ANCOVA). For comparison of baseline differences, the ANCOVA model included controls for investigator specialty. For end of study differences, controls included investigator specialty and baseline value, their interaction, and patient age. A chi-squared test was used to compare baseline differences in proportions of categorical variables. The Cochran-Mantel-Haenszel (CMH) test was used to compare baseline differences in proportions of ordered categorical variables. A one-sample, twotailed $t$-test was used to assess the significance of mean changes in continuous variables (KHQ domain scores and BDI-II summary score) within groups from baseline to study end. The kappa test of symmetry was used to assess the significance of changes from baseline in PPBC scores at 3 and 6 months among participants with postbaseline values. The kappa test of symmetry was also used to determine the significance of changes in individual KHQ questionnaire response items from baseline to study end; for display, response items were grouped as 'improved,' 'stayed the same' or 'worsened'. McNemar's test was used to calculate p-values for frequencies of paired data. CATI results were analysed with descriptive statistics.

\section{Results}

\section{Participants}

A total of 2888 individuals were enrolled at 327 study centres distributed throughout the continental USA (22). The analysis population consisted of all participants who were treated with OXY-TDS $(N=$ 2878) (22). A substantial number of men $(n=369$; $12.8 \%)$ participated, although most participants were female $(n=2508 ; 87.2 \%)$ (22). Demographic and baseline disease characteristics of male participants are presented in Table 1. Men in MATRIX were significantly $(\mathrm{p}<0.0001$; ANCOVA) older than female study participants (mean, 69.6 vs. 61.4 years); $71.3 \%$ of men $(n=263)$ were 65 years or older. Most men were Caucasian $(n=309 ; 83.7 \%)$, and about onequarter were employed $(n=87 ; 23.6 \%)$. OAB symptoms had started $\geq 4$ years ago in $39.3 \%$ of men. A majority of men $(n=207 ; 56.4 \%)$ had been treated at some time for OAB; the most common prior treatments were TOL-ER $(n=104 ; 28.2 \%)$, extended-release oxybutynin $(n=85 ; 23.0 \%)$, immediate-release tolterodine (TOL-IR; $n=46 ; 12.5 \%$ ) and OXY-IR $(n=42 ; 11.4 \%)$. Cardiovascular disease
Table 1 Demographic and baseline characteristics of male participants

\begin{tabular}{|c|c|}
\hline Characteristics & Men $(n=369)$ \\
\hline \multicolumn{2}{|l|}{ Age, years } \\
\hline Mean (standard deviation) & $69.6(13.1)$ \\
\hline Median (range) & $72.0(20-96)$ \\
\hline \multicolumn{2}{|l|}{ Race, $n(\%)$} \\
\hline White & $309(83.7)$ \\
\hline Black & $36(9.8)$ \\
\hline Asian & $10(2.7)$ \\
\hline Hispanic & $9(2.4)$ \\
\hline Others & $5(1.4)$ \\
\hline \multicolumn{2}{|l|}{ Employment status*, $n(\%)$} \\
\hline Employed $\dagger$ & $87(23.6)$ \\
\hline Not employed $\ddagger$ & $281(76.4)$ \\
\hline \multicolumn{2}{|c|}{ Comorbid conditions by body system, $n(\%)$} \\
\hline Cardiovascular & $245(66.4)$ \\
\hline Musculoskeletal & $169(45.8)$ \\
\hline Gastrointestinal & $138(37.4)$ \\
\hline Endocrine & $116(31.4)$ \\
\hline Neurological/psychiatric & $113(30.6)$ \\
\hline Renal & $82(22.2)$ \\
\hline Respiratory & $74(20.1)$ \\
\hline Dermatological & $50(13.6)$ \\
\hline Haematological/lymphatic & $24(6.5)$ \\
\hline Others & $204(55.3)$ \\
\hline \multicolumn{2}{|c|}{ Number of concurrent medications } \\
\hline Mean (standard deviation) & $5.8(4.7)$ \\
\hline Median (range) & $5(0-21)$ \\
\hline \multicolumn{2}{|c|}{$\begin{array}{l}\text { *One participant did not respond. †Working part time or full } \\
\text { time, with or without pay. } ¥ \text { Unemployed, disabled, retired or } \\
\text { other. }\end{array}$} \\
\hline
\end{tabular}

was the most common type of comorbidity $(n=$ 245 ; 66.4\%), and male participants most commonly used concomitant medications related to treatment of this condition: 147 (39.8\%) lipid-modifying agents, $139(37.7 \%)$ anti-thrombotic agents and 131 (35.5\%) agents that act on the renin-angiotensin system. Nearly all men $(n=320 ; 86.7 \%)$ were taking at least one concomitant medication.

About one-third of men (119/369; 32.2\%) had pre-existing prostate problems; of these, 67 were concurrently receiving BPH medication ( $\alpha$-blocker and/ or 5-ARI). Men with prostate problems were older than other male participants (mean age, 73.0 vs. 68.0 years; $\mathrm{p}=0.0010$; ANCOVA) but were similar in ethnicity ( $\mathrm{p}=0.4122$; chi-squared test $)$ and employment status $(\mathrm{p}=0.4783$; chi-squared test). The proportions of men with and without prostate problems were well balanced for diuretic use at baseline $(26.1 \%$ vs. $20.4 \%$; $p=0.2223$; chi-squared test). Time since onset of $\mathrm{OAB}$ symptoms was similar $(\mathrm{p}=0.3310$; $\mathrm{CMH}$ test $)$ in the two groups. A higher 
proportion of men with prostate problems (73 of 118 respondents; $61.9 \%$ ) than without (134 of 249 respondents; $53.8 \%$ ) had been treated previously for OAB; men with prostate problems (29/119; 24.4\%) were more likely than other men $(46 / 250 ; 18.4 \%)$ to have taken multiple $\mathrm{OAB}$ medications previously. More men with prostate problems than men without prostate problems had discontinued prior OAB treatments because of ineffectiveness $(61.2 \%$ vs. $56.3 \%$ respectively) and fewer because of compliance $(2.6 \%$ vs. $8.9 \%$ ), but other reasons for discontinuation of prior $\mathrm{OAB}$ therapies were given with similar fre- quency ('side effects', $21.6 \%$ vs. $19.8 \%$; other, $12.9 \%$ vs. $12.5 \%$; unknown, $1.7 \%$ vs. $2.6 \%$ ).

\section{Participant-reported perceptions of bladder condition}

At the baseline clinic visit, most male participants who responded to the PPBC (255/330; 77.3\%) reported a global $\mathrm{OAB}$ severity score of $\geq 4$ (Figure 1A). This percentage decreased sharply after 1 month of OXY-TDS treatment and continued on a declining trend for the remainder of the study (Figure 1A). At baseline, 16.5\% of male respondents

'Global assessment of $O A B$ severity' $\square \mathrm{PPBC}=1,2$ or 3 (less severe)

A

- $\mathrm{PPBC}=4,5$ or 6 (more severe)

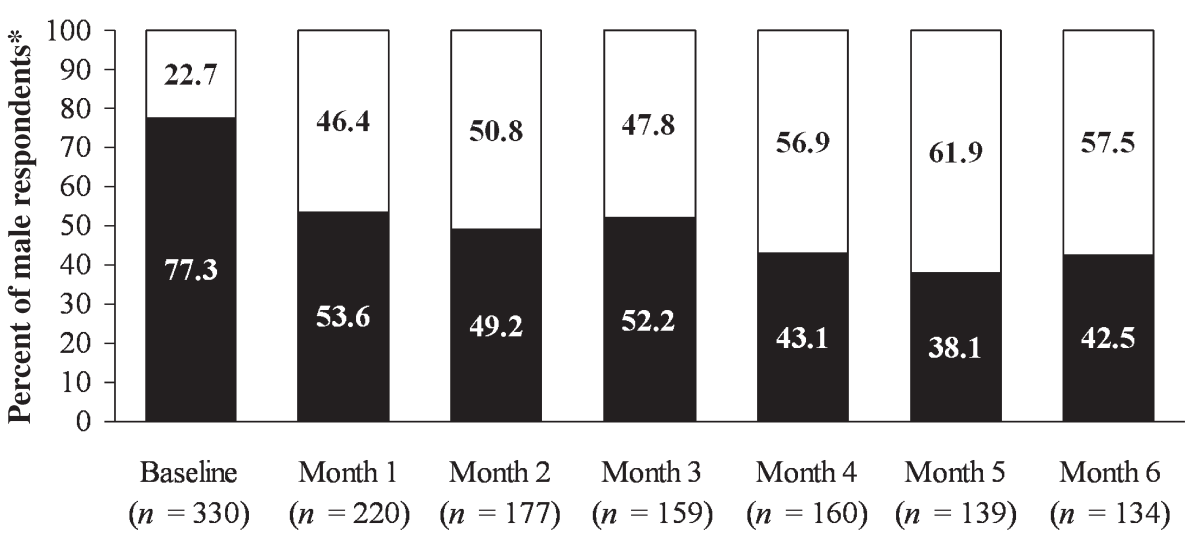

*Excludes participants who answered 'Do not know' or who refused to answer.

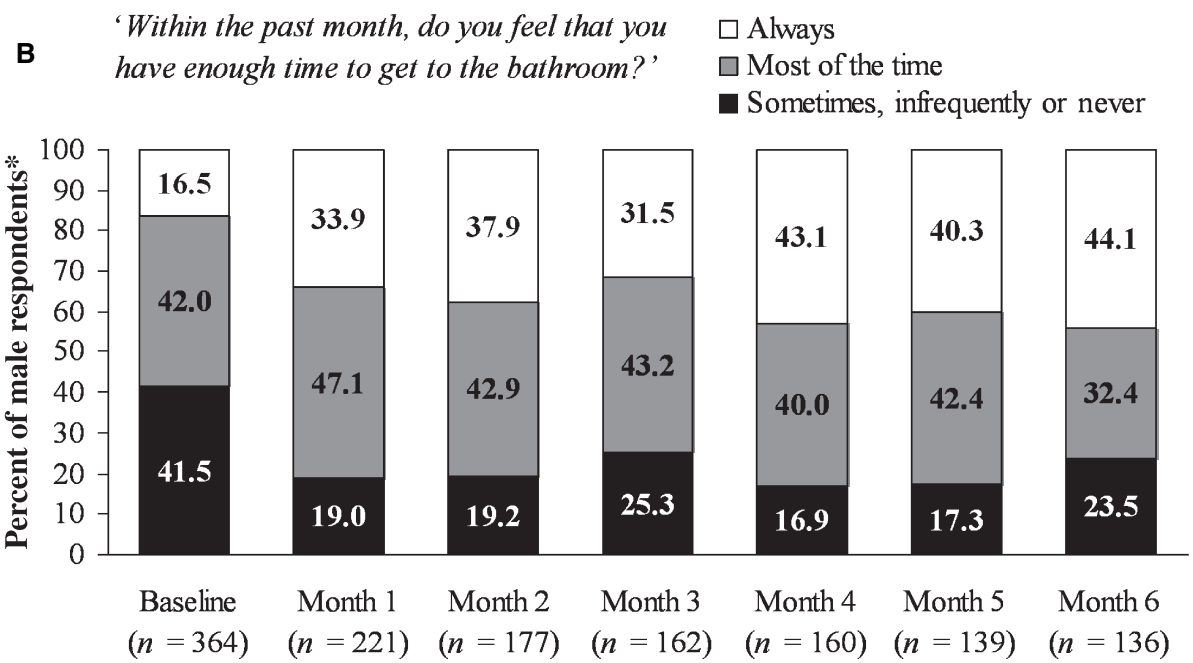

*Excludes participants who answered 'Do not know' or who refused to answer.

Figure 1 Participant-reported measures of bladder condition; percentages are calculated on the basis of the total number of respondents at each evaluation. (A) Percentage of respondents who rated their global overactive bladder (OAB) severity as 1 , 2 or 3 (no problems at all, very minor problems or some very minor problems) or 4, 5 or 6 (moderate problems, severe problems or many severe problems) with the Patient Perception of Bladder Condition (PPBC) questionnaire. (B) Percentage of respondents who reported feeling that they had enough time to get to the bathroom during the past month 
(60/364) felt that they 'always' had enough time to get to the bathroom (Figure 1B). After 1 month, the proportion who 'always' had enough time to get the bathroom had approximately doubled to $33.9 \%(75 / 221)$; the proportion was stable or increased slightly over the next 5 months (Figure 1B).

\section{$\mathrm{KHQ}$ response items and health-related quality of life}

At baseline, men in the study showed impairment of HRQoL in all 10 domains of the KHQ (Figure 2). The most impaired domains (highest scores) were Incontinence Impact, Symptom Severity, Sleep/ Energy and Physical Limitations (Figure 2). By study end, scores had improved in all KHQ domains, and changes were statistically significant ( $\mathrm{p} \leq 0.0196$; $t$-test) in eight of 10 domains (Figure 2). Changes were clinically meaningful in four of these eight domains: Incontinence Impact, Symptom Severity, Sleep/Energy and Role Limitations. The greatest absolute improvement was noted in the Incontinence
Impact domain (mean change of -8.9 points), and the greatest relative improvement was seen in the Role Limitations domain (mean change of $-21.0 \%$ ).

For 16 KHQ items, measured at baseline and study end, significantly more male participants reported improvement of symptoms than the number who reported worsening of symptoms (Figure 3). This was most evident in response items from the domains of Incontinence Impact, Symptom Severity, Role Limitations, Sleep/Energy and Severity (Coping) Measures (Figure 3). For the remaining KHQ items, no significant difference was noted in the number of participants who reported improvement or worsening of symptoms.

\section{Health-related quality of life in men with and without pre-existing prostate problems}

Men with and without pre-existing prostate problems had similar ( $\mathrm{p} \geq 0.1575$; ANCOVA) baseline scores in all $10 \mathrm{KHQ}$ domains. HRQoL improved in both groups from baseline to study end, as was shown by mean decreases in nine of $10 \mathrm{KHQ}$ domain scores

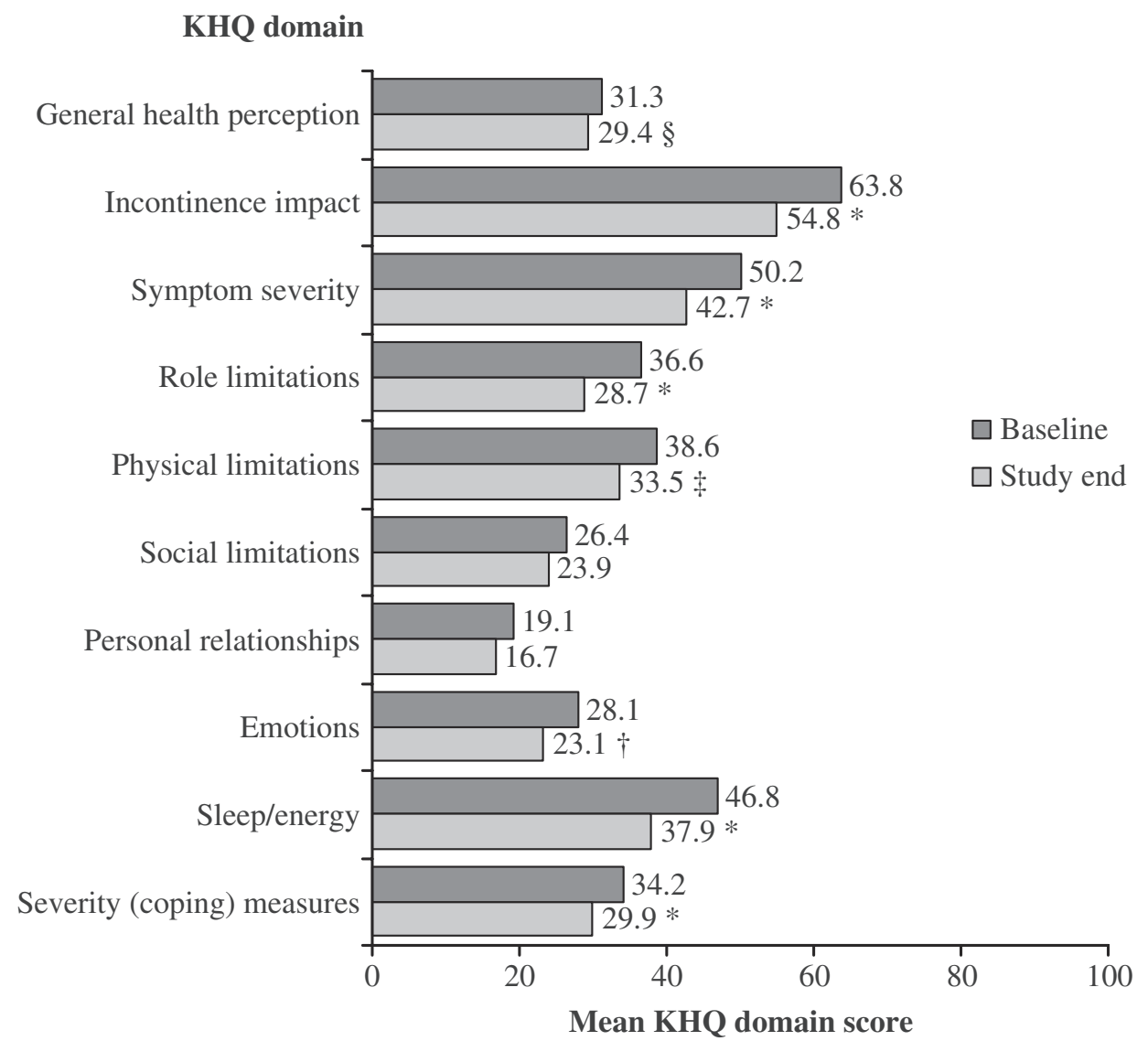

Figure 2 Health-related quality of life (HRQoL) of male participants at baseline and at study end, assessed as mean King's Health Questionnaire (KHQ) domain scores. ${ }^{*} \mathrm{p}<0.0001, \dagger \mathrm{p}<0.001$, $\ddagger \mathrm{p}<0.01, \$ \mathrm{p} \leq 0.0196$; one-sample $t$-test for significance of difference from zero for change in baseline value; other changes were not statistically significant $(\mathrm{p} \geq 0.1033)$ 


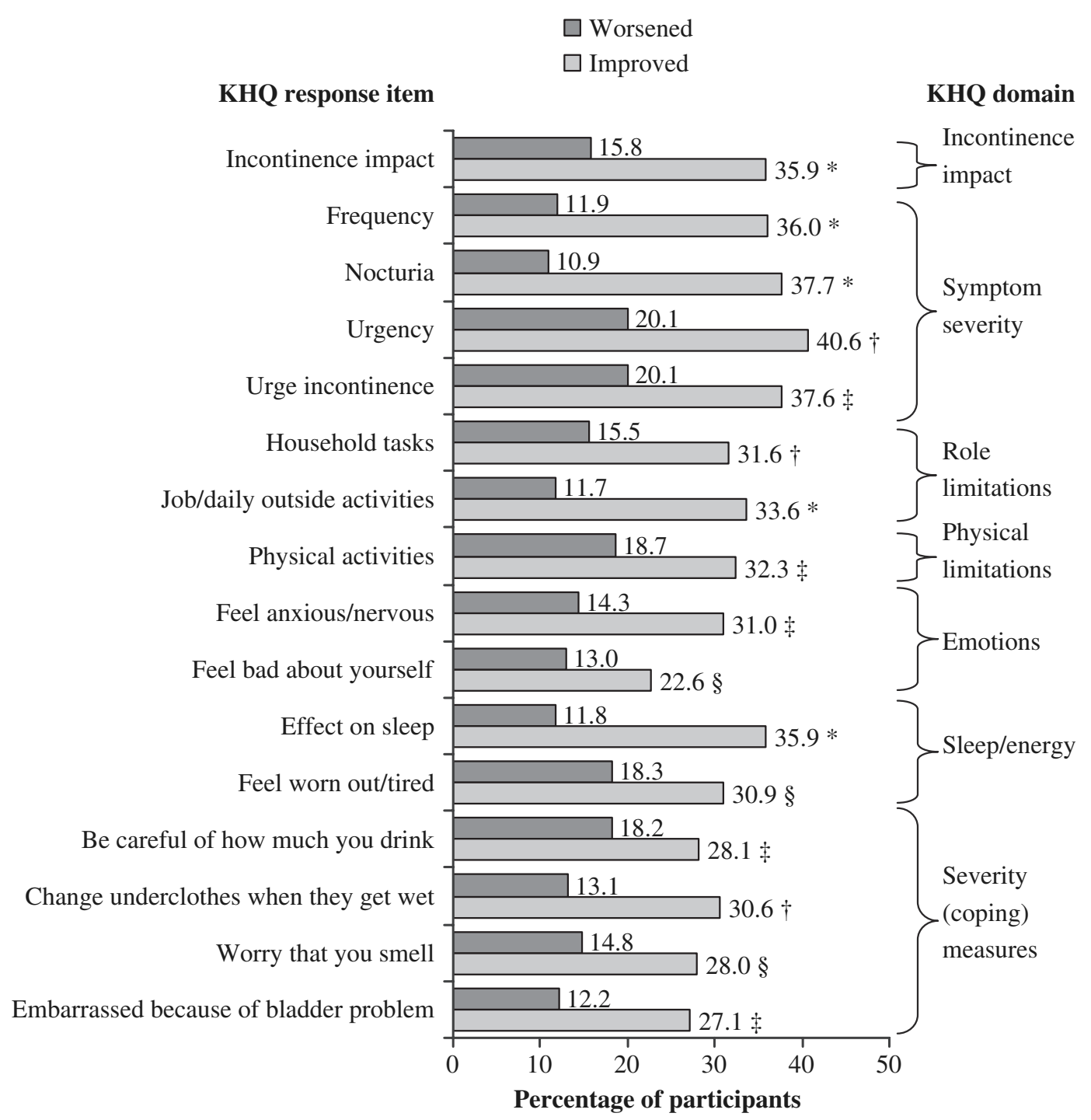

Figure 3 Percentage of male participants who reported improvement or worsening, from baseline to study end, in symptoms described in individual King's Health Questionnaire (KHQ) response items. Participants who reported that their symptoms 'stayed the same' are not depicted. Only responses for which statistically significant differences were noted are displayed. ${ }^{\star} \mathrm{p}<0.0001, \dagger \mathrm{p}<0.001, \ddagger \mathrm{p}<0.01, \S \mathrm{p} \leq 0.0488$; kappa test of symmetry; other comparisons were not significantly different $(\mathrm{p} \geq 0.1781)$

(Figure 4); changes were similar ( $\mathrm{p} \geq 0.1016$; ANCOVA) in all 10 domains for both groups.

\section{Symptoms of depression}

The mean BDI-II summary score at baseline for men in the study was 8.5 points; the median score was 7.0. By study end, the mean BDI-II summary score had improved significantly $(\mathrm{p}<0.0001 ; t$-test $)$ to 7.1 - a decrease of $16.5 \%$. Median BDI-II summary score decreased to 6.0 by study end. Among participants with postbaseline data, the proportion of men with a BDI-II summary score $>12$, which is associated with a diagnosis of clinical depression, decreased significantly, from $23.9 \%(72 / 301)$ at baseline to $17.9 \%(54 / 301)$ at study end $(\mathrm{p}=0.0055$; McNemar's test).

\section{Participant-reported satisfaction with treatment}

Most male participants (61.6-71.3\%) were 'very satisfied' or 'satisfied' with OXY-TDS throughout the study (Figure 5A). Conversely, a low proportion (8.6$15.4 \%$ ) were 'dissatisfied' or 'very dissatisfied' (Figure 5A). Similar results were obtained for questions about convenience, effectiveness and tolerability of the patch (data not shown). Most male participants who 
Men with prostate problems

Men without prostate problems

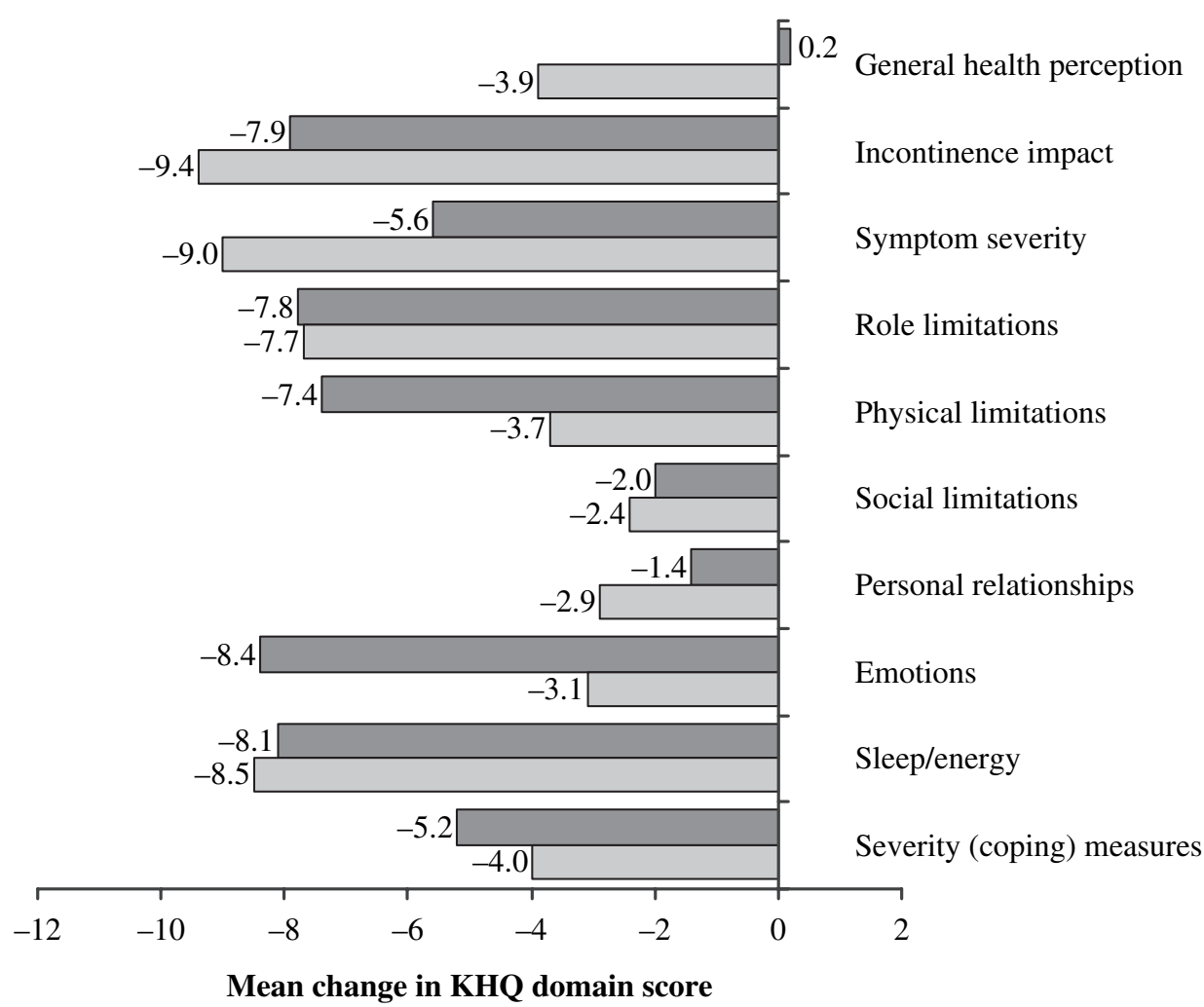

Figure 4 Improvement in health-related quality of life (HRQoL) in men with and without pre-existing prostate problems, assessed as mean changes in King's Health Questionnaire (KHQ) domain scores from baseline to study end. All changes in score were similar between the two groups ( $\mathrm{p} \geq 0.1016$ ); analysis of co-variance (ANCOVA)

had been treated previously for OAB (54.7-60.6\%) said that the patch offered significant or some benefits over previous treatments, suggesting that they preferred the patch over their prior therapy (Figure 5B). A small proportion preferred their previous medications $(7.2-12.8 \%)$. Similar opinions of the patch vs. prior therapy were reported with respect to ease of use, effectiveness and tolerability (data not shown).

\section{Safety}

Oxybutynin transdermal system was well tolerated; most men in the study $(281 / 369 ; 76.2 \%)$ experienced no drug-related adverse event during up to 6 months of treatment. The most common type of treatmentrelated adverse event, application site reaction, occurred in $13.8 \%(51 / 369)$ of male participants (Table 2). The vast majority of these reactions $(71 / 73$; $97.3 \%$ ) were judged mild or moderate in severity. Treatment-related anticholinergic adverse events were infrequently reported by male participants: dry mouth $(n=9 ; 2.4 \%)$, nausea $(n=5 ; 1.4 \%)$, headache $(n=$ $4 ; 1.1 \%)$, constipation $(n=4 ; 1.1 \%)$, dizziness $(n=$ $3 ; 0.8 \%)$, abdominal pain $(n=2 ; 0.5 \%)$, dry eye $(n=1 ; 0.3 \%)$ and dysuria $(n=1 ; 0.3 \%)$. The male participant who experienced treatment-related mild dysuria continued study treatment after a 2-day hiatus. A total of 47 men (12.7\%) discontinued study participation because of a treatment-related adverse event. No male participants experienced a serious treatment-related adverse event.

Two participants $(2 / 369 ; 0.5 \%)$ from the male cohort each reported one event of urinary retention, both of which were considered related to treatment. However, these events were mild in severity and neither required catheterisation. One participant who experienced urinary retention continued treatment with OXY-TDS, and the other discontinued treatment. Male and female study participants had comparable rates of urinary retention [2/369 men $(0.5 \%)$ vs. $8 / 2508$ women $(0.3 \%)]$ and dysuria [1/369 men $(0.3 \%)$ vs. $14 / 2508$ women $(0.6 \%)]$.

\section{Discussion}

This planned subgroup analysis of MATRIX participants was among the longest-term, single, prospec- 


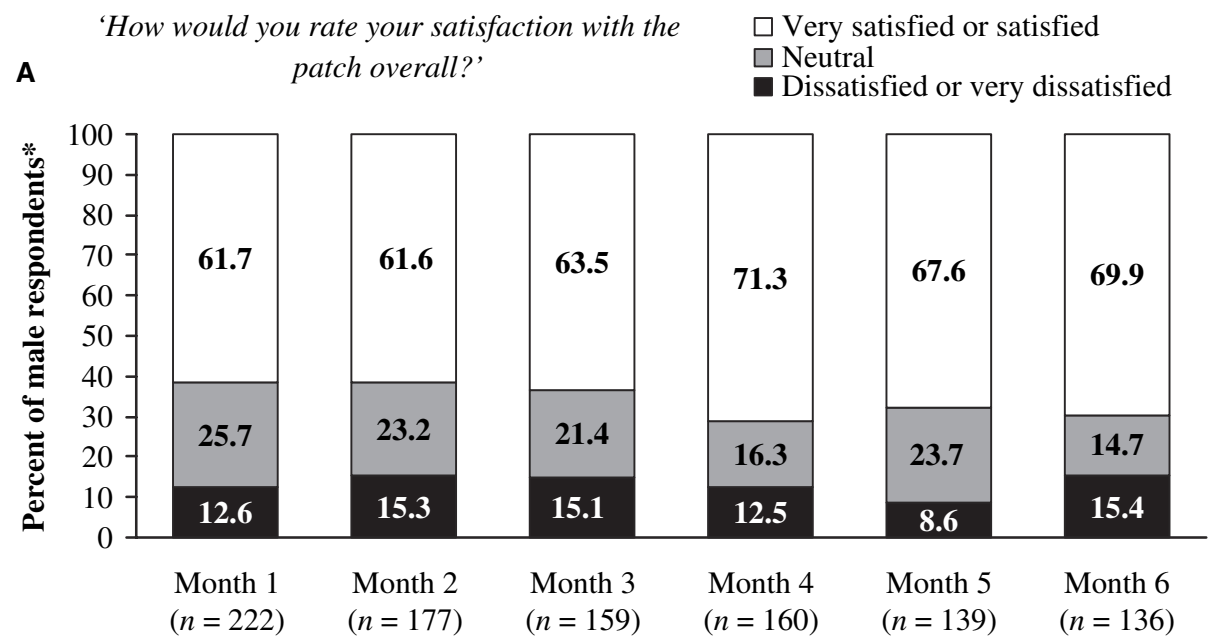

*Excludes participants who answered 'N/A' or who had missing data.

B 'How does the patch compare with your previous treatment for overactive bladder overall?'

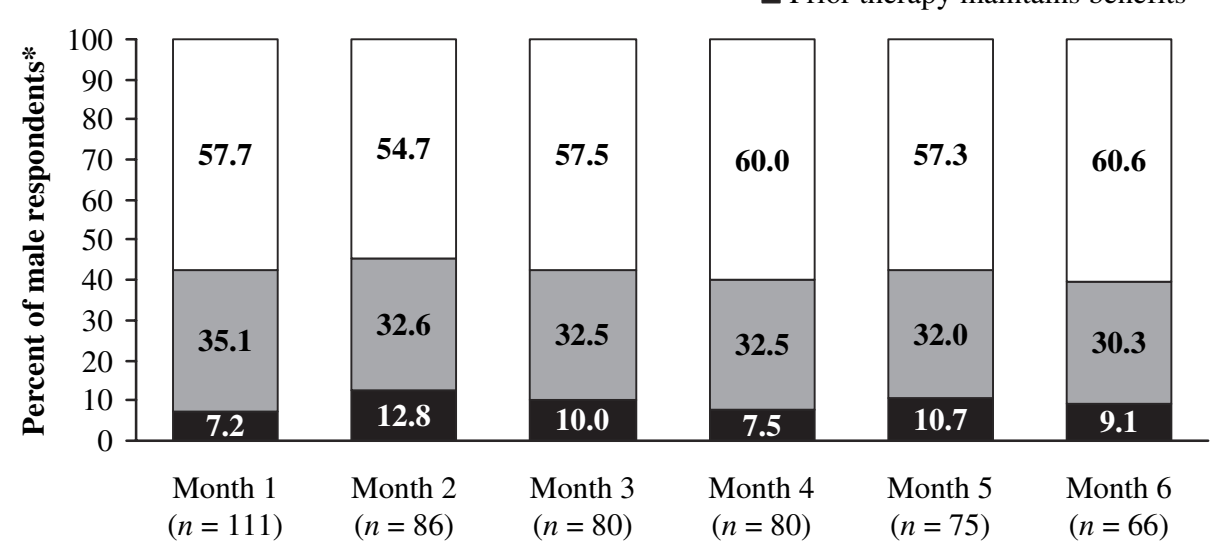

$\square$ Patch offers benefits

$\square$ Neutral

Prior therapy maintains benefits

*Excludes participants who answered 'N/A' or who had missing data.

Figure 5 Participant-reported satisfaction with oxybutynin transdermal system (OXY-TDS) treatment; percentages are calculated on the basis of the total number of respondents at each evaluation. (A) Overall satisfaction in the total population of male participants. (B) Overall satisfaction in male participants who had been treated before for overactive bladder $(\mathrm{OAB})$

tive assessments of the effectiveness of antimuscarinic treatment for $\mathrm{OAB}$ in a large cohort of men. As intended, the study population was representative of actual patients with $\mathrm{OAB}$ in terms of advanced age, comorbidity and use of concomitant medications. Many study participants had not persisted with previous $\mathrm{OAB}$ treatments, which also is a common characteristic of patients with $\mathrm{OAB}$ in the general population (12). MATRIX was the first study of men with $\mathrm{OAB}$ to have a patient-reported outcome (i.e. change in HRQoL) as its primary end-point. Concurrent use of the PPBC allowed comparison of participant-reported changes in global OAB severity, independent of the symptoms and HRQoL changes measured with the KHQ. This study did not have a placebo or active control; therefore, results cannot determine causal relationships. However, the openlabel design was closer to clinical practice than blinded treatment. The large population size, minimally restrictive entry criteria and 6-month duration of the study also allowed better estimation of the incidence of relatively rare adverse events.

Global OAB severity in male participants, as selfassessed with the PPBC, improved distinctly from baseline to month 1 and continued to improve slightly during the remaining 5 months. Perceived urinary urgency (not enough 'time to get to the bathroom'), which is a defining symptom of $\mathrm{OAB}$, 
Table 2 Treatment-related adverse events occurring in $\geq 1 \%$ of male participants

\begin{tabular}{lc}
\hline Adverse event & Male participants, \\
\hline Application site reaction* & $\boldsymbol{n}(\%)$ \\
Dry mouth & $51(13.8)$ \\
Pruritus & $9(2.4)$ \\
Erythema & $8(2.2)$ \\
Nausea & $5(1.4)$ \\
Constipation & $5(1.4)$ \\
Headache & $4(1.1)$ \\
Rash & $4(1.1)$ \\
Somnolence & $4(1.1)$ \\
\end{tabular}

*A participant may have reported more than one type of application site (AS) reaction. Types included AS pruritus (22; $6.0 \%)$, AS dermatitis $(20 ; 5.4 \%)$, AS erythema $(15 ; 4.1 \%)$, AS irritation $(6 ; 1.6 \%)$, AS pain $(2 ; 0.5 \%)$, AS vesicles $(2$; $0.5 \%)$, AS bleeding $(1 ; 0.3 \%)$, AS burning $(1 ; 0.3 \%)$ and AS swelling $(1 ; 0.3 \%)$.

also improved markedly and within the same time as global $\mathrm{OAB}$ severity. This perceived change in urinary urgency is consistent with the significant ( $p<0.001$; kappa test of symmetry) improvement noted in the KHQ 'urgency' response item. Other symptoms and HRQoL items recorded with the KHQ also improved significantly during the study. These results are similar to those from a pooled analysis of two studies in men with $\mathrm{OAB}$, in which treatment with TOL-ER significantly improved daytime and 24-h micturitions, although not nighttime micturitions (29). In MATRIX, treatment with OXY-TDS was associated with significant ( $\mathrm{p}<0.0001$; kappa test of symmetry) improvement in urinary frequency and nocturia, as assessed through KHQ response items. The degree of HRQoL improvement in MATRIX was unaffected by preexisting prostate conditions or the concomitant use of medications to treat $\mathrm{BPH}$. These results are consistent with those of a randomised, controlled study of men with $\mathrm{OAB}$ and other LUTS, in which significantly more patients $(172 / 215 ; 80 \%$; $p<0.001)$ treated with TOL-ER and an $\alpha$-blocker (tamsulosin) reported treatment benefit, compared with patients who received placebo $(132 / 214 ; 62 \%)(15)$.

Depression symptoms monitored with the BDI-II decreased significantly during the study for men treated with OXY-TDS. The KHQ response item that asked participants whether they 'Feel bad about yourself also showed significant ( $\mathrm{p}<0.05$; kappa test of symmetry) improvement from baseline to study end. No other studies have shown improvement in depression among men being treated for OAB.
Indeed, little evidence to date suggests that treatment improves depression symptoms in any population of patients with $\mathrm{OAB}$, despite evidence that depression symptoms are common in these individuals (4). Kelleher et al. found no significant effect of TOL-ER or TOL-IR vs. placebo on the SF-36 Mental Summary score $(7,30)$.

The number of male participants who were 'satisfied' or 'very satisfied' with OXY-TDS overall was four to eightfold greater, at various times during the study, than the number who reported being 'dissatisfied' or 'very dissatisfied.' For men with $\mathrm{OAB}$ who had been previously treated, the ratio of 'satisfied/very satisfied' to 'dissatisfied/very dissatisfied' also ranged from four- to eightfold at different times during the study. Taken together, these results suggest that perceived effectiveness, as well as convenience and perceived tolerability, was associated with treatment satisfaction in male participants.

Oxybutynin transdermal system was well tolerated by the male cohort in MATRIX and no serious adverse events were considered related to treatment. The rate of application site reactions in men (13.8\%) was similar to that observed in the overall MATRIX population (14.0\%) and in previous studies $(17,18,20,22)$. A low incidence of anticholinergic adverse events was observed, despite the use of concomitant medications by the great majority of men. Despite some suggestion from another study that tolterodine and tamsulosin may have a synergistic effect on the incidence of dry mouth, in MATRIX, dry mouth did not occur significantly more frequently among men who took $\alpha$-blockers than among those who did not (3/56 vs. 6/307; p = 0.142; Fisher's exact test) (15). No cases of acute urinary retention requiring catheterisation were reported in the male cohort described here, which was followed for up to 6 months. This low probability of urinary retention observed in individuals with $\mathrm{BPH}$ or other prostate problems is consistent with results from studies of TOL-ER treatment in patients with $\mathrm{OAB}$, some of whom also had BOO or additional LUTS $(15,29,31)$.

The International Continence Society definition of $\mathrm{OAB}$ is based on symptoms of urinary urgency, frequency and incontinence (32). Validated questionnaires that assess changes in these symptoms are important measures for improvement in $\mathrm{OAB}$ severity (33). The European Agency for the Evaluation of Medicinal Products has stated that 'the primary aim for developing new drugs for UI should be to obtain a subjective improvement or cure of symptoms for the patient' (34). In male study participants, perceived $\mathrm{OAB}$ severity, $\mathrm{OAB}$ symptoms and HRQoL all improved with OXY-TDS treatment. 
Serious adverse effects related to treatment were not observed during the study; urinary retention was rare $(0.5 \%$ of all male participants $)$ and resolved without the need for catheterisation. Satisfaction with treatment was good, even among those who had discontinued prior therapies. Participants in the present analysis were typical of $\mathrm{OAB}$ patients encountered in general practice in terms of their advanced age, multiple comorbid conditions and use of many concomitant medications. The use of questionnaires in general medical practice could allow effective treatment of many patients without the need for specialised urodynamic evaluations.

Although the design of MATRIX was appropriate for its goal of evaluating therapeutic success in a large, clinically relevant population, some inherent limitations should be noted. The study did not include objective assessments of response, such as urodynamic measurements or micturition frequency, but instead relied on participant-reported outcomes. It is not known how well symptoms recorded with the KHQ relate to symptoms measured with instruments that are commonly used to evaluate LUTS in men, such as the IPSS or the American Urological Association Symptom Score. However, evidence suggests a correlation between KHQ and IPSS results in Japanese patients $(35,36)$. The post hoc analysis by history of prostate problems or concomitant $\mathrm{BPH}$ medication use was retrospective; therefore, selection bias may have influenced the results. Nonetheless, the positive outcomes reported in men who had a history of prostate problems or who were using $\mathrm{BPH}$ medications suggest that this last study limitation was of little consequence. In fact, a primary aim of MATRIX was to include individuals, such as these, who might be commonly encountered in clinical practice, but who have been excluded from nearly all randomised, controlled clinical trials conducted previously.

In summary, men with $\mathrm{OAB}$ reported improved OAB severity, HRQoL and depression symptoms after treatment with OXY-TDS. Treatment effects were similar in men with or without pre-existing prostate problems. Participant-reported perceptions of OXY-TDS supported the more objective measures of effectiveness and showed a high degree of satisfaction with treatment. OXY-TDS was well tolerated in men, none of whom experienced acute urinary retention or a treatment-related serious adverse event during up to 6 months of treatment. OXY-TDS appears to be a viable treatment for men with LUTS, many of whom have $\mathrm{OAB}$ alone or together with $\mathrm{BPH} /$ BPO (37).

\section{Acknowledgements}

The authors acknowledge Scientific Connexions, Newtown, Pennsylvania, USA, for providing editorial assistance in the preparation of this manuscript. Funding support was provided by Watson Laboratories, Inc., Morristown, New Jersey, USA. The sponsor designed the MATRIX study, conducted the data analysis, and reviewed this manuscript prior to submission.

\section{Author contributions}

David R. Staskin: Study design, data analysis and manuscript preparation. Matt T. Rosenberg: Study investigator and manuscript preparation. Naomi $V$. Dahl: Study design, data analysis and manuscript preparation. Paul V. Polishuk: Study investigator and manuscript preparation. Norman R. Zinner: Study investigator and manuscript preparation.

\section{References}

1 Milsom I, Abrams P, Cardozo L et al. How widespread are the symptoms of an overactive bladder and how are they managed? A population-based prevalence study. BJU Int 2001; 87: 760-6.

2 Stewart WF, Van Rooyen JB, Cundiff GW et al. Prevalence and burden of overactive bladder in the United States. World J Urol 2003; 20: 327-36.

3 Berry SJ, Coffey DS, Walsh PC, Ewing LL. The development of human benign prostatic hyperplasia with age. J Urol 1984; 132: 474-9.

4 Irwin DE, Milsom I, Kopp Z et al. Impact of overactive bladder symptoms on employment, social interactions and emotional wellbeing in six European countries. BJU Int 2006; 97: 96-100.

5 Temml C, Heidler S, Ponholzer A, Madersbacher S. Prevalence of the overactive bladder syndrome by applying the International Continence Society definition. Eur Urol 2005; 48: 622-7.

6 Zakaria L, Anastasiadis AG, Shabsigh R. Common conditions of the aging male: erectile dysfunction, benign prostatic hyperplasia, cardiovascular disease and depression. Int Urol Nephrol 2001; 33: 283-92.

7 Kelleher CJ, Reese PR, Pleil AM, Okano GJ. Health-related quality of life of patients receiving extended-release tolterodine for overactive bladder. Am J Manag Care 2002; 8: S608-15.

8 Kelleher CJ, Cardozo L, Chapple CR et al. Improved quality of life in patients with overactive bladder symptoms treated with solifenacin. BJU Int 2005; 95: 81-5.

9 Roehrborn CG, Abrams P, Rovner ES et al. Efficacy and tolerability of tolterodine extended-release in men with overactive bladder and urgency urinary incontinence. BJU Int 2006; 97: 1003-6.

10 Kaplan SA, Roehrborn CG, Dmochowski $\mathrm{R}$ et al. Tolterodine extended release improves overactive bladder symptoms in men with overactive bladder and nocturia. Urology 2006; 68: 328-32.

11 Jumadilova Z, Harris H, del Aguila M et al. Agent Selection for Overactive Bladder Patients with and without Documented Comorbid Benign Prostatic Hyperplasia. Presented at the International Continence Society 35th Annual Meeting. Montreal, QC, 2005.

12 Yu YF, Nichol MB, Yu AP, Ahn J. Persistence and adherence of medications for chronic overactive bladder/urinary incontinence in the California Medicaid Program. Value Health 2005; 8: $495-$ 505 . 
13 Herbison P, Hay-Smith J, Ellis G, Moore K. Effectiveness of anticholinergic drugs compared with placebo in the treatment of overactive bladder: systematic review. BMJ 2003; 326: 841-7.

14 McConnell J, Abrams P, Khoury S et al. for the International Scientific Committee. (2005) Evaluation and treatment of lower urinary tract symptoms (LUTS) in older men. In: McConnell S, Abrams P, Denis L et al., eds. Proceedings from the 6th International Consultation on New Developments in Prostate Cancer and Prostate Diseases, 24-27 June. Paris, France: Health Publications, pp. 387-401.

15 Kaplan SA, Roehrborn CG, Rovner ES et al. Tolterodine and tamsulosin for treatment of men with lower urinary tract symptoms and overactive bladder: a randomized controlled trial. JAMA 2006 296: 2319-28.

16 Lee JY, Kim HW, Lee SJ et al. Comparison of doxazosin with or without tolterodine in men with symptomatic bladder outlet obstruction and an overactive bladder. BJU Int 2004; 94: 817-20.

17 Davila GW, Daugherty CA, Sanders SW, for the Transdermal Oxybutynin Study Group. A short-term, multicenter, randomized double-blind dose titration study of the efficacy and anticholinergic side effects of transdermal compared to immediate release oral oxybutynin treatment of patients with urge urinary incontinence. J Urol 2001; 166: 140-5.

18 Dmochowski RR, Sand PK, Zinner NR et al. for the Transdermal Oxybutynin Study Group. Comparative efficacy and safety of transdermal oxybutynin and oral tolterodine versus placebo in previously treated patients with urge and mixed urinary incontinence. Urology 2003; 62: 237-42.

19 Appell RA, Chancellor MB, Zobrist RH et al. Pharmacokinetics, metabolism, and saliva output during transdermal and extendedrelease oral oxybutynin administration in healthy subjects. Mayo Clin Proc 2003; 78: 696-702.

20 Dmochowski RR, Davila GW, Zinner NR et al. for the Transdermal Oxybutynin Study Group. Efficacy and safety of transdermal oxybutynin in patients with urge and mixed urinary incontinence. J Urol 2002; 168: 580-6.

21 Dmochowski RR, Nitti V, Staskin D et al. Transdermal oxybutynin in the treatment of adults with overactive bladder: combined results of two randomized clinical trials. World J Urol 2005; 23: 263-70.

22 Sand P, Zinner N, Newman D et al. Oxybutynin transdermal system improves the quality of life in adults with overactive bladder: a multicentre, community-based, randomized study. BJU Int 2007; 99: $836-44$.

23 Sand PK, Goldberg RP, Dmochowski RR et al. The impact of the overactive bladder syndrome on sexual function: a preliminary report from the Multicenter Assessment of TRansdermal therapy In overactive bladder with oXybutynin trial. Am J Obstet Gynecol 2006; 195: 1730-5.

24 Coyne KS, Matza LS, Kopp Z, Abrams P. The validation of the Patient Perception of Bladder Condition (PPBC): a single-item global measure for patients with overactive bladder. Eur Urol 2006; 49: 1079-86.

25 Kelleher CJ, Cardozo LD, Khullar V, Salvatore S. A new questionnaire to assess the quality of life of urinary incontinent women. Br J Obstet Gynaecol 1997; 104: 1374-9.

26 Kobelt G, Kirchberger I, Malone-Lee J. Quality-of-life aspects of the overactive bladder and the effect of treatment with tolterodine. BJU Int 1999; 83: 583-90.

27 Kelleher CJ, Pleil AM, Reese PR et al. How much is enough and who says so? The case of the King's Health Questionnaire and overactive bladder. BJOG 2004; 111: 605-12.

28 Beck AT, Ward $\mathrm{CH}$, Mendelson $\mathrm{M}$ et al. An inventory for measuring depression. Arch Gen Psychiatry 1961; 4: 561-71.

29 Dmochowski R, Abrams P, Marschall-Kehrel D et al. Efficacy and tolerability of tolterodine extended release in male and female patients with overactive bladder. Eur Urol 2007; 51: 1054-64.

30 Pleil AM, Reese PR, Kelleher CJ, Okano GJ. Health-related quality of life of patients with overactive bladder receiving immediaterelease tolterodine. Health Econ Prev Care 2001; 2: 69-75.

31 Abrams P, Kaplan S, De Koning Gans HJ, Millard R. Safety and tolerability of tolterodine for the treatment of overactive bladder in men with bladder outlet obstruction. J Urol 2006; 175: 9991004

32 Abrams P, Cardozo L, Fall M et al. for the Standardisation Subcommittee of the International Continence Society. The standardisation of terminology of lower urinary tract function: report from the Standardisation Sub-committee of the International Continence Society. Neurourol Urodyn 2002; 21: 167-78.

33 Brubaker L, Chapple C, Coyne KS, Kopp Z. Patient-reported outcomes in overactive bladder: importance for determining clinical effectiveness of treatment. Urology 2006; 68 (Suppl. 2A): 3-8.

34 European Agency for the Evaluation of Medicinal Products and Committee for Proprietary Medicinal Products. Note for Guidance on the Clinical Investigation of Medicinal Products for the Treatment of Urinary Incontinence. London, UK, 2002. http://www.emea. europa.eu/pdfs/human/ewp/001801en.pdf (accessed 3 August 2007).

35 Okamura K, Usami T, Nagahama K, Maruyama S, Mizuta E. 'Quality of life' assessment of urination in elderly Japanese men and women with some medical problems using International Prostate Symptom Score and King's Health Questionnaire. Eur Urol 2002; 41: 411-9.

36 Okamura K, Usami T, Nagahama K, Maruyama S, Mizuta E. The relationships among filling, voiding subscores from International Prostate Symptom Score and quality of life in Japanese elderly men and women. Eur Urol 2002; 42: 498-505.

37 Rosenberg MT, Staskin DR, Kaplan SA et al. A practical guide to the evaluation and treatment of male lower urinary tract symptoms in the primary care setting. Int J Clin Pract 2007; 61: 153546 (OnlineEarly Articles).

Paper received September 2007, accepted October 2007 